\title{
SPECTRAL THEORY OF FUNCTIONS IN STUDYING PARTIAL DIFFERENTIAL OPERATORS
}

\author{
A.G. BASKAKOV, E.E. DIKAREV
}

\begin{abstract}
The work is devoted to studying the spectral properties of differential operators with constant coefficients defined on subspaces of bounded continuous functions. Our main methods are spectral theory of Banach modules, theory of functions, abstract harmonic analysis and theory of representations, which were developed and described in detail in the monograph by A. G. Baskakov "Harmonic Analysis in Banach Modules and the Spectral Theory of Linear Operators", Voronezh, VSU Publ., 2016. We introduce the algebra of polynomials by means of which we define differential operators. We also introduce closed subspaces of the space of bounded continuous functions called homogeneous function spaces, which play an important role in the analysis. An important class of spectrally homogeneous spaces is introduced as well. We obtain results relating the zero set of a polynomial with the properties of kernels and images of differential operators defined by these polynomials. We define the notion of a regular at infinity polynomial (ellipticity-type conditions) and we provide important examples of partial differential operators defined by such polynomials. The conditions of invertibility of such differential operators are obtained. In particular, we obtain criteria of invertibility in spectrally homogeneous spaces and spaces of periodic function. We get a result on coincidence of the spectrum of a differential operator with the image of polynomial generating this operator in spectrally homogeneous spaces. Conditions of compactness of the resolvent of partial differential operators defined by polynomials regular at the infinity are found.
\end{abstract}

Keywords: patrial differential operator, regular polynomial, Beurling spectrum of function, spectrum of operator, Banach module, kernel and image of linear operator, invertibility of operator

Mathematics Subject Classification: 47B38+42B35

\section{INTRODUCTION AND MAIN RESULTS}

We consider an algebra $\mathcal{P}=\mathcal{P}\left(\mathbb{R}^{N}\right)$ of polynomials of form

$$
p: \mathbb{R}^{N} \rightarrow \mathbb{C}, \quad p(\xi)=\sum_{|\alpha| \leqslant m} a_{\alpha} \xi^{\alpha}, \quad a_{\alpha} \in \mathbb{C}, \alpha \in \mathbb{Z}_{+}^{N}, \xi \in \mathbb{R}^{N}
$$

where $\xi^{\alpha}=\xi_{1}^{\alpha_{1}} \ldots \xi_{N}^{\alpha_{N}}, \xi=\left(\xi_{1}, \ldots, \xi_{N}\right) \in \mathbb{R}^{N}, \alpha=\left(\alpha_{1}, \ldots, \alpha_{N}\right) \in \mathbb{Z}_{+}^{N}, \mathbb{Z}_{+}=\mathbb{N} \cup\{0\}$, $m, N \in \mathbb{N}$.

By means of the polynomials in the algebra $\mathcal{P}\left(\mathbb{R}^{N}\right)$ we introduce differential operators acting in subspaces of the Banach space $\mathrm{C}_{\mathrm{b}}=\mathrm{C}_{\mathrm{b}}\left(\mathbb{R}^{N}\right)$ of continuous and bounded on $\mathbb{R}^{N}$ functions

A.G. Baskakov, E.E. Dikarev, Spectral theory of Functions in Studying Partial Differential OPERATORS.

(C)Baskakov A.G., Dikarev E.E. 2019.

The reported study of the first author was funded by RFBR according to the research project no. 19-0100732. The reported study of the second author was funded by RFBR according to the research project no. 18-31-00354.

Submitted August 10, 2017. 
equipped with the norm $\|x\|=\sup _{\tau \in \mathbb{R}^{N}}|x(\tau)|$. We study their spectral properties: we describe the properties of the kernel, image and the spectrum and obtain the invertibility conditions.

The present work was essentially motivated by the paper by E. Mukhamadiev, A.N. Naimov, A.Kh. Sattorov [1], in which there were obtained the necessary and sufficient conditions for the invertibility in $\mathrm{C}_{\mathrm{b}}$ of the differential operators constructed by a certain class of polynomials described in Example 3 after Definition 7.

The definition of the operators and the study of their spectral properties relies essentially on the spectral theory of function and the spectral theory of Banach moduli. This is why first we recall some employed notions and results from works [2], [3], [4], [5], 6].

By the symbol $\mathrm{L}^{1}=\mathrm{L}^{1}\left(\mathbb{R}^{N}\right)$ we denote the Banach algebra of summable on $\mathbb{R}^{N}$ classes of measurable complex functions with the convolution of the function

$$
(f * g)(\tau)=\int_{\mathbb{R}^{N}} f(\tau-s) g(s) \mathrm{d} s, \quad \tau \in \mathbb{R}^{N}, f, g \in \mathrm{L}^{1}\left(\mathbb{R}^{N}\right),
$$

as the multiplication and wit the norm $\|f\|_{1}=\int_{\mathbb{R}^{N}}|f(\tau)| \mathrm{d} \tau, \quad f \in \mathrm{L}^{1}\left(\mathbb{R}^{N}\right)$.

The symbol $\widehat{f}: \mathbb{R}^{N} \rightarrow \mathbb{C}$ stands for the Fourier transform

$$
\widehat{f}(\lambda)=\int_{\mathbb{R}^{N}} f(\tau) \mathrm{e}^{-\mathrm{i}(\lambda, \tau)} \mathrm{d} \lambda, \quad \lambda=\left(\lambda_{1}, \ldots, \lambda_{N}\right) \in \mathbb{R}^{N},
$$

of a function $f \in \mathrm{L}^{1}\left(\mathbb{R}^{N}\right)$. By $(\lambda, \tau)$ we denote the scalar product of vectors $\lambda, \tau \in \mathbb{R}^{N}$.

The algebra of Fourier transforms with the pointwise multiplication of the functions in $\mathrm{L}^{1}\left(\mathbb{R}^{N}\right)$ is denoted by $\widehat{\mathrm{L}^{1}}\left(\mathbb{R}^{N}\right)$.

Definition 1. A complex Banach space $\mathcal{X}$ is called a Banach $\mathrm{L}^{1}\left(\mathbb{R}^{N}\right)$ modulus if there defined a bilinear mapping

$$
(f, x) \mapsto f x: \mathrm{L}^{1}\left(\mathbb{R}^{N}\right) \times X \rightarrow X
$$

with the following properties:

1) $(f * g) x=f(g x), f, g \in \mathrm{L}^{1}\left(\mathbb{R}^{N}\right), x \in X$;

2) $\|f x\| \leqslant\|f\|_{1}\|x\|, f \in \mathrm{L}^{1}\left(\mathbb{R}^{N}\right), x \in X$;

3) if $f x=0$ for each $f \in \mathrm{L}^{1}\left(\mathbb{R}^{N}\right)$, then $x=0$ (a non-degenerate property of the modulus).

In what follows, by End $\mathcal{X}$ we denote a Banach algebra of linear bounded operators (endomorphisms) acting in the complex Banach space $\mathcal{X}$.

Usually the structure of a Banach $\mathrm{L}^{1}\left(\mathbb{R}^{N}\right)$-modulus on the Banach space $\mathcal{X}$ is defined by means of some strongly continuous isometric representation $T: \mathbb{R}^{N} \rightarrow$ End $\mathcal{X}$ by the following formula

$$
f x=\int_{\mathbb{R}^{N}} f(\tau) T(-\tau) x \mathrm{~d} \tau, \quad f \in \mathrm{L}^{1}, x \in X .
$$

The Banach space $\mathrm{C}_{\mathrm{b}}\left(\mathbb{R}^{N}\right)$ is a Banach $\mathrm{L}^{1}\left(\mathbb{R}^{N}\right)$-modulus, whose structure is defined by the convolution of the functions

$$
(f * x)(\tau)=\int_{\mathbb{R}^{N}} f(s) x(\tau-s) \mathrm{d} s=\int_{\mathbb{R}^{N}} f(\tau-s) x(s) \mathrm{d} s, \quad \tau \in \mathbb{R}^{N}, f \in \mathrm{L}^{1}, x \in \mathrm{C}_{\mathrm{b}} .
$$

We mention the estimate $\|f * x\| \leqslant\|f\|_{1}\|x\|$.

In the Banach space $\mathrm{C}_{\mathrm{b}}=\mathrm{C}_{\mathrm{b}}\left(\mathbb{R}^{N}\right)$ we consider the group of isometries

$$
S: \mathbb{R}^{N} \rightarrow \text { End } \mathrm{C}_{\mathrm{b}}, \quad(S(\tau) x)(s)=x(s+\tau), \quad s, \tau \in \mathbb{R}^{N}, x \in \mathrm{C}_{\mathrm{b}} .
$$


Despite this group is not strongly continuous, nevertheless, formula (2) is reduced to formula (3) for $T=S$ (here $\mathcal{X}=\mathrm{C}_{\mathrm{b}}$ ).

Differential operators are defined on Banach spaces of functions by the following definition.

Definition 2. A non-zero closed subspace $\mathcal{F}=\mathcal{F}\left(\mathbb{R}^{N}\right)$ in $\mathrm{C}_{\mathrm{b}}=\mathrm{C}_{\mathrm{b}}\left(\mathbb{R}^{N}\right)$ is called a homogeneous space of continuous functions if the following conditions hold:

1) the subspace $\mathcal{F}$ is invariant with respect to the operators $S(\tau), \tau \in \mathbb{R}^{N}$;

2) $\mathcal{F}$ is a submodulus in a $\mathrm{L}^{1}\left(\mathbb{R}^{N}\right)$-modulus $\mathrm{C}_{\mathrm{b}}\left(\mathbb{R}^{N}\right)$, that is, $f * x \in \mathcal{F}$ for each function $f \in \mathrm{L}^{1}\left(\mathbb{R}^{N}\right), x \in \mathcal{F}$.

The following closed subspaces in $\mathrm{C}_{\mathrm{b}}\left(\mathbb{R}^{N}\right)$ are homogeneous.

1. The subspace $\mathrm{C}_{\mathrm{b}, \mathrm{u}}=\mathrm{C}_{\mathrm{b}, \mathrm{u}}\left(\mathbb{R}^{N}\right)$ of uniformly continuous functions in $\mathrm{C}_{\mathrm{b}}\left(\mathbb{R}^{N}\right)$.

2. The subspace $\mathrm{C}_{0}=\mathrm{C}_{0}\left(\mathbb{R}^{N}\right) \subset \mathrm{C}_{\mathrm{b}, \mathrm{u}}$ of functions vanishing at infinity.

3. The subspace $\mathrm{C}_{\mathrm{sl}, \infty}=\mathrm{C}_{\mathrm{sl}, \infty}\left(\mathbb{R}^{N}\right) \subset \mathrm{C}_{\mathrm{bu}}$ of slowly varying functions: $x \in \mathrm{C}_{\mathrm{sl}, \infty}$ ig $\lim _{|\tau| \rightarrow \infty}\|S(\tau) x-x\|=0$, where $|\tau|=\left|\tau_{1}\right|+\cdots+\left|\tau_{N}\right|$ for each $\tau \in \mathbb{R}^{N}$.

4. The subspace $\mathrm{C}_{\Omega}=\mathrm{C}_{\Omega}\left(\mathbb{R}^{N}\right)$ of periodic functions in $\mathrm{C}_{\mathrm{b}}$ with the group of periods $\Omega \subset \mathbb{R}^{N}$; the factor group $\mathbb{R}^{N} / \Omega$ is isomorphic to $\omega \mathbb{Z}^{N}$ with $\left.\omega=\left(\omega_{1}, \ldots, \omega_{N}\right) \in \mathbb{R}_{+}^{N}, \mathbb{R}_{+}=(0, \infty)\right)$.

5. The subspace $\mathrm{AP}=\operatorname{AP}\left(\mathbb{R}^{N}\right)$ of Bohr almost periodic functions (see [7], [8]): $\operatorname{AP}\left(\mathbb{R}^{N}\right)=$ $\overline{\operatorname{span}}\left\{\mathrm{e}_{\lambda} \mid \lambda \in \mathbb{R}^{N}\right\}$, where $\mathrm{e}_{\lambda}(\tau)=\mathrm{e}^{\mathrm{i}(\lambda, \tau)}, \tau \in \mathbb{R}^{N}$.

6. The subspace $\mathrm{AP}_{\infty}=\mathrm{AP}_{\infty}\left(\mathbb{R}^{N}\right)$ of almost periodic at infinity functions (see [9], [10], [11]) defined by the identity

$$
\mathrm{AP}_{\infty}=\overline{\operatorname{span}}\left\{\mathrm{e}_{\lambda}(\tau) x \mid \lambda \in \mathbb{R}^{N}, x \in \mathrm{C}_{\mathrm{sl}, \infty}\right\} .
$$

Other spaces of homogeneous functions form spectral subspaces, see Definition 6. We note that in all homogeneous spaces the norm is induced from $\mathrm{C}_{\mathrm{b}}\left(\mathbb{R}^{N}\right)$.

Definition 3. A homogeneous space of functions $\mathcal{F}$ is called spectrally homogeneous if all functions $x \mathrm{e}_{\lambda}, \lambda \in \mathbb{R}^{N}, x \in \mathcal{F}$, belong to $\mathcal{F}\left(\mathbb{R}^{N}\right)$.

All aforementioned examples of homogeneous spaces apart of the space of periodic functions $\mathrm{C}_{\Omega}$ and the space $\mathrm{C}_{\mathrm{sl}, \infty}$ are spectrally homogeneous.

We proceed to constructing differential operators in some homogeneous space of functions $\mathcal{F}=\mathcal{F}\left(\mathbb{R}^{N}\right)$. In order to do this, we consider a sublagebra $\mathcal{M}$ of the functions in the algebra $\mathrm{L}^{1}\left(\mathbb{R}^{N}\right)$ whose Fourier transform is compactly supported. If $f \in \mathcal{M}$, it follows from the formula of the inverse Fourier transform

$$
f(\tau)=\frac{1}{(2 \pi)^{N}} \int_{\mathbb{R}^{N}} \widehat{f}(\lambda) \mathrm{e}^{\mathrm{i}(\lambda, \tau)} \mathrm{d} \lambda=\frac{1}{(2 \pi)^{N}} \int_{V} \widehat{f}(\lambda) \mathrm{e}^{\mathrm{i}(\lambda, \tau)} \mathrm{d} \lambda, \quad \tau \in \mathbb{R}^{N},
$$

where $V$ is a compact neighbourhood in $\mathbb{R}^{N}$ containing the support supp $\widehat{f}$ of the function $\widehat{f}$, that the subalgebra $\mathcal{M}$ possesses the following properties:

1) Each function $f \in \mathcal{M}$ possesses all partial derivatives

$$
\mathbb{D}_{k} f, \quad 1 \leqslant k \leqslant N, \quad \mathbb{D}^{\alpha} f=(-1)^{|\alpha|} \mathbb{D}_{1}^{\alpha_{1}} \ldots \mathbb{D}_{N}^{\alpha_{N}} f, \quad \alpha=\left(\alpha_{1}, \ldots, \alpha_{N}\right) \in \mathbb{Z}_{+}^{N},
$$

and they all belong both to the Banach algebra $\mathrm{L}^{1}\left(\mathbb{R}^{N}\right)$ and the algebra $\mathrm{C}_{0}\left(\mathbb{R}^{N}\right)$;

2) Each function $f: \mathbb{R}^{N} \rightarrow \mathbb{C}$ in $\mathcal{M}$ admits an extension on $\mathbb{C}$ to the functions of a finite exponential type [12];

3) The subalgebra $\mathcal{M}$ is dense in $\mathrm{L}^{1}\left(\mathbb{R}^{N}\right)$.

Thus, the operators

$$
\mathbb{D}^{\alpha}=\mathbb{D}_{1}^{\alpha_{1}} \ldots \mathbb{D}_{N}^{\alpha_{N}}: \mathcal{M} \rightarrow \mathcal{M}, \quad \alpha \in \mathbb{Z}_{+}^{N},
$$


are well-defined on the algebra $\mathcal{M}$ and they are continuous in the topology of the Schwarz space.

We consider an arbitrary polynomial $p \in \mathcal{P}\left(\mathbb{R}^{N}\right)$ of form (1) and for each $f \in \mathcal{M}$ and $x \in \mathcal{F}$ we let

$$
\mathcal{A}_{p}(f * x)=p(f) * x,
$$

where $p(f)=\sum_{|\alpha| \leqslant m} a_{\alpha} \mathbb{D}^{\alpha} f$ is a function in the algebra $\mathcal{M}$.

Definition 4. A differential operator $\mathcal{A}_{p}: \mathrm{D}\left(\mathcal{A}_{p}\right) \subset \mathcal{F} \rightarrow \mathcal{F}$ is defined as follows. A function $x \in \mathcal{F}$ is in the domain $\mathrm{D}\left(\mathcal{A}_{p}\right)$ of the operator $\mathcal{A}_{p}$ if there exists a function $y \in \mathcal{F}$ such that for each function $f \in \mathcal{M}$ the identities hold:

$$
\sum_{|\alpha| \leqslant m} a_{\alpha} \mathbb{D}^{\alpha}(f * x)=p(f) * x=f * y, \quad p(f)=\sum_{|\alpha| \leqslant m} a_{\alpha} \mathbb{D}^{\alpha} f \in \mathrm{L}^{1}\left(\mathbb{R}^{N}\right) .
$$

We observe that the operator $\mathcal{A}_{p}$ is well-defined since the function $y$ is determined uniquely by the function $x \in \mathcal{F}$. Otherwise, if a function $x$ would have been mapped by this rule into two functions $y, z \in \mathcal{F}$, the identity $f *(y-z)=0$ had been satisfied for each function $f \in \mathcal{M}$. Therefore, since $\mathcal{M}$ is dense in the algebra $\mathrm{L}^{1}\left(\mathbb{R}^{N}\right)$, we obtain that $y-z=0$.

The constructed operator $\mathcal{A}_{p}: \mathrm{D}\left(\mathcal{A}_{p}\right) \subset \mathcal{F} \rightarrow \mathcal{F}$ will be often denoted shortly by $\mathcal{A}_{p, \mathcal{F}}$. His linearity is implied by its well-defined property. A close but not coinciding definition was given in [13].

Lemma 1. The operator $\mathcal{A}_{p, \mathcal{F}}$ is closed and commutes with the convolution operators $S(f) x=f * x, x \in \mathcal{F}, f \in \mathrm{L}^{1}\left(\mathbb{R}^{N}\right)$, as well as with the operators $S(\tau), \tau \in \mathbb{R}^{N}$, of translation of functions in $\mathcal{F}$.

We observe that the commuting of the linear operator $A: \mathrm{D}(A) \subset X \rightarrow X$ and a bounded operator $B \in$ End $\mathcal{X}$, where $\mathcal{X}$ is a Banach space, means that $B \mathrm{D}(A) \subset \mathrm{D}(A)$ and the identities $A B x=x, x \in X, B A y=y, y \in \mathrm{D}(A)$, hold.

While defining other homogeneous spaces and proving the main results we shall employ the Beurling spectrum of the vectors in Banach $\mathrm{L}^{1}\left(\mathbb{R}^{N}\right)$-moduli and its properties, see [2], [3], 14], [15], [16], [17], [18]. As such homogeneous spaces, the spectral subspaces in the homogeneous space $\mathcal{F}$ will serve, see Definition 2 .

Let $\mathcal{X}$ be a Banach $\mathrm{L}^{1}\left(\mathbb{R}^{N}\right)$-modulus.

Definition 5. Beurling spectrum of a vector $x \in X$ is the set of the elements in $\mathbb{R}^{N}$ of the form

$$
\begin{aligned}
\Lambda(x)= & \left\{\lambda_{0} \in \mathbb{R}^{N} \mid f x \neq 0 \text { for each function } f \in \mathrm{L}^{1}\left(\mathbb{R}^{N}\right)\right. \text {, satisfying } \\
& \left.\widehat{f}\left(\lambda_{0}\right) \neq 0\right\}=\mathbb{R}^{N} \backslash\left\{\mu_{0} \in \mathbb{R}^{N} \mid \text { there exists a function } f \in \mathrm{L}^{1}\left(\mathbb{R}^{N}\right)\right. \\
& \text { such that } \left.\widehat{f}\left(\mu_{0}\right) \neq 0 \text { and } f x=0\right\} .
\end{aligned}
$$

Beurling spectrum of a subset $X_{0}$ in $X$ is the set in $\mathbb{R}^{N}$ of the form

$$
\begin{aligned}
\Lambda\left(X_{0}\right)= & \overline{\bigcup_{x \in X_{0}} \Lambda(x)}=\mathbb{R}^{N} \backslash\left\{\mu_{0} \in \mathbb{R}^{N} \mid \text { there exists a function } f \in \mathrm{L}^{1}\left(\mathbb{R}^{N}\right)\right. \\
& \text { such that } \left.\widehat{f}\left(\mu_{0}\right) \neq 0 \text { and } f x=0 \text { for each vector } x \text { in } X_{0}\right\} .
\end{aligned}
$$

The Beurling spectrum $\Lambda(x)$ of each function $x \in \mathrm{C}_{\mathrm{b}}$ coincides with the support of the Fourier transform of a function $x$ regarded as a distribution [19], [20], [21, [22]. For instance, the Beurling spectrum $\Lambda(x)$ of almost each periodic function $x \in \operatorname{AP}\left(\mathbb{R}^{N}\right)$ coincides with the Bohr spectrum of the function $x$, see [2], [3]. This property is employing in the proof of Theorem 7. 
Definition 6. For each closed set $\Delta$ in $\mathbb{R}^{N}$, the subspace $X(\Delta)$ in $X$ of the form

$$
X(\Delta)=\{x \in \mathcal{X} \mid \Lambda(x) \subset \Delta\}
$$

is called a spectral submodulus in a $\mathrm{L}^{1}\left(\mathbb{R}^{N}\right)$-modulus $\mathcal{X}$. If $\mathcal{X}=\mathcal{F}$, then the spectral submodulus $\mathcal{F}(\Delta)$ is called spectral subspace.

We note that $\Lambda(X(\Delta)) \subset \Delta$ but not necessary the identity. At that we observe that each internal point $\lambda_{0} \in \operatorname{Int} \Delta$ is contained in $\Lambda(X(\Delta))$.

Lemma 2. For each vector $x$ in the domain $\mathrm{D}\left(\mathcal{A}_{p, \mathcal{F}}\right)$ of the operator $\mathcal{A}_{p, \mathcal{F}}$ the inclusion of the spectra

$$
\Lambda\left(\mathcal{A}_{p, \mathcal{F}} x\right) \subset \Lambda(x), \quad x \in \mathrm{D}\left(\mathcal{A}_{p, \mathcal{F}}\right)
$$

holds true.

For each polynomial $p$ in $\mathcal{P}\left(\mathbb{R}^{N}\right)$, by the symbol $\mathrm{Z}(p)$ we denote the set of its zeroes:

$$
\mathrm{Z}(p)=\left\{\lambda \in \mathbb{R}^{N} \mid p(\lambda)=0\right\} .
$$

The following statements on the kernel of the operator $\mathcal{A}_{p, \mathcal{F}}$ hold.

Theorem 1. The kernel $\operatorname{Ker} \mathcal{A}_{p, \mathcal{F}}=\left\{x \in \mathrm{D}\left(\mathcal{A}_{p, \mathcal{F}}\right) \mid \mathcal{A}_{p, \mathcal{F}} x=0\right\}$ is contained in the spectral subspace $\mathcal{F}(\Delta)$, where $\Delta=\mathrm{Z}(p)$, and is a closed submodulus in $\mathcal{F}$ invariant with respect to the operators $S(\tau), \tau \in \mathbb{R}^{N}$.

Theorem 2. Let the set $\mathrm{Z}(p)$ of zeroes of a polynomial $p$ is at most countable and $\mathrm{D}\left(\mathcal{A}_{p, \mathcal{F}}\right) \subset$ $\mathrm{C}_{\mathrm{b}, \mathrm{u}}\left(\mathrm{R}^{N}\right)$; the latter means that the functions in the domain are uniformly continuous. Then the following properties hold:

1) $\operatorname{Ker} \mathcal{A}_{p, \mathcal{F}} \subset \operatorname{AP}\left(\mathbb{R}^{N}\right)$, that is, the functions in the kernel Ker $\mathcal{A}_{p, \mathcal{F}}$ are Bohr almost continuous;

2) $\operatorname{Ker} \mathcal{A}_{p, \mathcal{F}}$ is a separable subspace in $\mathrm{C}_{\mathrm{b}, \mathrm{u}}$;

3) $\operatorname{Ker} \mathcal{A}_{p, \mathcal{F}}=\{0\}$, that is, the operator $\mathcal{A}_{p, \mathcal{F}}$ is injective if $\mathcal{F}=\mathrm{C}_{0}$;

4) the kernel of the operator $\mathcal{A}_{p, \mathcal{F}}$ is finite-dimensional if the set $\mathrm{Z}(p)$ is finite; at that, it consists of trigonometric polynomials;

5) $\mathcal{A}_{p, \mathcal{F}}$ is a injective operator if $\mathrm{Z}(p)$ is the empty set.

In the next two theorems we formulate the properties of the image $\operatorname{Im} \mathcal{A}_{p, \mathcal{F}}$ of the operator $\mathcal{A}_{p, \mathcal{F}}$.

Theorem 3. The subspace $\operatorname{Im} \mathcal{A}_{p, \mathcal{F}}$ is a submodulus (not necessarily closed) in $\mathcal{F}$ invariant with respect to the operators $S(\tau), \tau \in \mathbb{R}^{N}$.

Theorem 4. The image $\operatorname{Im} \mathcal{A}_{p, \mathcal{F}}$ of the operator $\mathcal{A}_{p, \mathcal{F}}$ possesses the following properties:

1) $\operatorname{Im} \mathcal{A}_{p, \mathcal{F}}$ contains a spectral subspace $\mathcal{F}(\Delta)$ for each compact set $\Delta \subset \mathbb{R}^{N}$ with the property $\Delta \cap \mathrm{Z}(p)=\varnothing$

2) $\operatorname{Im} \mathcal{A}_{p, \mathcal{F}}$ is a dense in $\mathcal{F}$ subspace if $\mathcal{F} \subset \mathrm{C}_{\mathrm{b}, \mathrm{u}}$ and $\mathrm{Z}(p)=\varnothing$;

3) $\operatorname{Im} \mathcal{A}_{p, \mathrm{C}_{0}}$ (here $\mathcal{F}=\mathrm{C}_{0}$ ) is a dense in $\mathrm{C}_{0}$ subspace if the set $\mathrm{Z}(p)$ contains no finite limiting points;

4) $\operatorname{Im} \mathcal{A}_{p, \mathcal{F}} \subset \mathrm{C}_{0}$ if $\mathcal{F}=\mathrm{C}_{\mathrm{sl}}$ and $p(0)=0$.

While obtaining the conditions of invertibility of the operator $\mathcal{A}_{p, \mathcal{F}}$ and especially the criterions for a non-empty resolvent set $\rho\left(\mathcal{A}_{p, \mathcal{F}}\right)$ of this operator, we are led to restrictions for the behavior of the polynomial at infinity. The conditions given below are motivated by the aim to represent the inverse operator as a convolution with some function in the algebra $L^{1}\left(\mathbb{R}^{N}\right)$. 
Definition 7. The polynomial $p \in \mathcal{P}\left(\mathbb{R}^{N}\right)$ is called regular at infinity if there exists a number $R \geqslant 0$ and a pair of functions $f_{1}, f_{0}$ in the algebra $\mathrm{L}^{1}\left(\mathbb{R}^{N}\right)$ such that

$$
p(\lambda) \widehat{f}_{1}(\lambda)-\widehat{f}_{0}(\lambda)=1
$$

for all vectors $\lambda=\left(\lambda_{1}, \ldots, \lambda_{N}\right) \in \mathbb{R}^{N}$ obeying the condition $|\lambda|=\left|\lambda_{1}\right|+\cdots+\left|\lambda_{N}\right| \geqslant R$.

The set of regular at infinity polynomials is denoted by the symbol $\mathcal{P}_{\text {reg, } \infty}\left(\mathbb{R}^{N}\right)$.

We note that the polynomials defining elliptic operators are regular at infinity, see, for instance, [21, Ch. 2, Sect. 12].

Let us provide more specific examples of regular at infinity polynomials:

1) $p_{1}(\xi)=\xi_{1}+\mathrm{i} \xi_{2}, \xi=\left(\xi_{1}, \xi_{2}\right) \in \mathbb{R}^{2}, p_{1} \in \mathcal{P}\left(\mathbb{R}^{2}\right)$;

2) $p_{2}(\xi)=\sum_{k=1}^{N} \xi_{k}^{2}, \xi=\left(\xi_{1}, \ldots, \xi_{N}\right) \in \mathbb{R}^{N}, p_{2} \in \mathcal{P}\left(\mathbb{R}^{N}\right)$;

3) $p_{3}(\xi)=\prod_{k=1}^{N} p_{k}\left(\xi_{k}\right)+\sum_{\left|\alpha_{k}\right| \leqslant m_{k}-1} a_{\alpha} \xi^{\alpha}$, where $p_{k}: \mathbb{R} \rightarrow \mathbb{C}, 1 \leqslant k \leqslant N$, are polynomials of degree $m_{k}, \sum_{k=1}^{m} m_{k}=N$ and $\alpha=\left(\alpha_{1}, \ldots, \alpha_{N}\right) \in \mathbb{Z}_{+}^{N}$.

The first polynomial generates the operator $\mathcal{A}_{p_{1}}=\frac{\partial}{\partial \xi_{1}}+\mathrm{i} \frac{\partial}{\partial \xi_{2}}$; the second does the Laplace operator $\mathcal{A}_{p_{2}}=\Delta$, and the third polynomial was considered in paper [1] while constructing and studying the invertibility conditions of the corresponding differential operator $\mathcal{A}_{p_{3}}$.

Theorem 5. Let $p \in \mathcal{P}_{\text {reg, },}\left(\mathbb{R}^{N}\right)$. The operator $\mathcal{A}_{p}: \mathrm{D}\left(\mathcal{A}_{p}\right) \subset \mathcal{F} \rightarrow \mathcal{F}$ is invertible if and only if the condition

$$
\mathrm{Z}(p) \cap \Lambda(\mathcal{F})=\varnothing
$$

holds true, where $\Lambda(\mathcal{F})$ is the Beurling spectrum of a $\mathrm{L}^{1}\left(\mathbb{R}^{N}\right)$-modulus $\mathcal{F}$. If condition (8) is satisfied, the inverse operator $\mathcal{A}_{p}^{-1}$ admits the representation

$$
\mathcal{A}_{p}^{-1} y=g * y, \quad y \in \mathcal{F},
$$

for some function $g \in \mathrm{L}^{1}\left(\mathbb{R}^{N}\right)$.

In view of the identity $\Lambda(\mathcal{F})=\mathbb{R}^{N}$ for a spectrally homogeneous space $\mathcal{F}$, see Remark 2 , Theorem 5 implies immediately the following statement.

Theorem 6. Let $p: \mathbb{R}^{N} \rightarrow \mathbb{C}$ be a regular at infinity polynomial and $\mathcal{F}$ be a non-zero spectrally homogeneous space. The operator $\mathcal{A}_{p, \mathcal{F}}$ is invertible if and only if

$$
\mathrm{Z}(p)=\varnothing \text {. }
$$

If the latter holds, the operator inverse for $\mathcal{A}_{p, \mathcal{F}}$ admits representation (9), where the Fourier transform of the function $g$ reads as

$$
\widehat{g}(\lambda)=\frac{1}{p(\lambda)}, \quad \lambda \in \mathbb{R}^{N}
$$

In the proof of the next theorem we shall employ the identity [2], [3]:

$$
\begin{aligned}
\Lambda\left(\mathrm{C}_{\Omega}\right)=\left\{2 \pi\left(\frac{k_{1}}{\omega_{1}}, \ldots, \frac{k_{N}}{\omega_{N}}\right) \mid k\right. & =\left(k_{1}, \ldots, k_{N}\right) \in \mathbb{Z}^{N}, \\
\omega & \left.=\left(\omega_{1}, \ldots, \omega_{N}\right) \in \mathbb{R}_{+}^{N}, \text { is a set in the group of periods } \Omega\right\} .
\end{aligned}
$$


Theorem 7. If $p \in \mathcal{P}_{\text {reg, }, \infty}\left(\mathbb{R}^{N}\right)$, then the operator $\mathcal{A}_{p}: \mathrm{D}\left(\mathcal{A}_{p}\right) \subset \mathrm{C}_{\Omega} \rightarrow \mathrm{C}_{\Omega}=\mathrm{C}_{\Omega}\left(\mathbb{R}^{N}\right)$ is invertible if and only if the condition

$$
\mathrm{Z}(p) \cap\left\{2 \pi\left(\frac{k_{1}}{\omega_{1}}, \ldots, \frac{k_{N}}{\omega_{N}}\right) \mid k=\left(k_{1}, \ldots, k_{N}\right) \in \mathbb{Z}^{N}\right\}=\varnothing
$$

holds true. Under condition (10), the inverse operator is defined by the formula

$$
\left(\mathcal{A}_{p}^{-1} y\right)(\tau)=\int_{K} G(\tau-s) y(s) \mathrm{d} s,
$$

where $K=\left[0, \omega_{1}\right] \times \cdots \times\left[0, \omega_{N}\right]$ is a compact set and $\Phi \in \mathrm{L}^{1}(K), G: K \rightarrow \mathbb{C}$ is a summable on $K$ function with a Fourier series of the form

$$
G(\tau) \sim \sum_{k \in \mathbb{Z}^{N}} \frac{1}{p\left(2 \pi\left(\frac{k_{1}}{\omega_{1}}, \ldots, \frac{k_{N}}{\omega_{N}}\right)\right)} \mathrm{e}^{\mathrm{i} 2 \pi(k, \widetilde{\omega})}, \quad \tau \in \mathbb{R}^{N},
$$

where $\widetilde{\omega}=\left(\frac{1}{\omega_{1}}, \ldots, \frac{1}{\omega_{N}}\right)$.

Theorem 8. For $p \in \mathcal{P}_{\text {reg, }, \infty}\left(\mathbb{R}^{N}\right)$ the spectrum $\sigma\left(\mathcal{A}_{p, \mathcal{F}}\right)$ of the operator $\mathcal{A}_{p, \mathcal{F}}$ is represented as

$$
\sigma\left(\mathcal{A}_{p, \mathcal{F}}\right)=p(\Lambda(\mathcal{F}))=\{p(\lambda) \mid \lambda \in \Lambda(\mathcal{F})\} .
$$

If $\mathcal{F}$ is a spectrally homogeneous space, then

$$
\sigma\left(\mathcal{A}_{p, \mathcal{F}}\right)=\operatorname{Im} p=p\left(\mathbb{R}^{N}\right) .
$$

Theorem 9. Let $p \in \mathcal{P}_{\text {reg, } \infty}\left(\mathbb{R}^{N}\right)$ and the Beurling spectrum $\Lambda(\mathcal{F})$ of a homogeneous space $\mathcal{F}$ has no finite limiting points in $\mathbb{R}^{N}$. Then the operator $\mathcal{A}_{p, \mathcal{F}}$ has a compact resolvent.

\section{Harmonic analysis in Banach moduli over algebra $L^{1}\left(\mathbb{R}^{N}\right)$}

Let $X$ be a Banach $\mathrm{L}^{1}\left(\mathbb{R}^{N}\right)$-modulus and $T: \mathbb{R}^{N} \rightarrow$ End $\mathcal{X}$ is an isometric representation not necessarily strongly continuous.

Definition 8. We shall say that the structure of a Banach $\mathrm{L}^{1}(\mathbb{R})-$ modulus $\mathcal{X}$ is associated with a representation $T$ if for all $f \in \mathrm{L}^{1}\left(\mathbb{R}^{N}\right), x \in \mathcal{X}$ and $\tau \in \mathbb{R}^{N}$ the identities hold

$$
T(\tau)(f x)=(S(\tau) f) x=f(T(\tau) x), \quad \tau \in \mathbb{R}^{N} .
$$

For such modulus $\mathcal{X}$ we employ the symbol $(\mathcal{X}, T)$.

In particular, the structure of a Banach $\mathrm{L}^{1}\left(\mathbb{R}^{N}\right)$-modulus on $\mathrm{C}_{\mathrm{b}}\left(\mathbb{R}^{N}\right)$ is associated with the representation $S: \mathbb{R}^{N} \rightarrow$ End $\mathrm{C}_{\mathrm{b}}$.

The vectors in a Banach $\mathrm{L}^{1}\left(\mathbb{R}^{N}\right)$-modulus $(X, T)$ have the following properties, see [2], 3], [14], [15], [16], [17]). The symbol $T(f)$ stands for the operator $T(f) \in$ End $X, T(f) x=f x$, $x \in X$, where $f \in \mathrm{L}^{1}\left(\mathbb{R}^{N}\right)$ is an arbitrary function. Thus, $S(f)$ is a convolution operator in each homogeneous space $\mathcal{F}$.

Lemma 3. The Beurling spectrum of the vectors in Banach $\mathrm{L}^{1}\left(\mathbb{R}^{N}\right)$-modulus $\mathcal{X}$ possess the following properties

1) $\Lambda(x)$ is a closed set for each vector $x \in X$ and $\Lambda(x)=\varnothing$ if and only if $x=0$;

2) $\Lambda\left(\mathcal{B}_{1} x_{1}+\mathcal{B}_{2} x_{2}\right) \subset \Lambda\left(x_{1}\right) \cup \Lambda\left(x_{2}\right)$ for each two operators $\mathcal{B}_{1}, \mathcal{B}_{1} \in$ End $\mathcal{X}$ commuting with the operators $T(f), f \in \mathrm{L}^{1}\left(\mathbb{R}^{N}\right)$, and for all vectors $x_{1}, x_{2} \in X$;

3) $\Lambda(f x) \subset(\operatorname{supp} \widehat{f}) \cap \Lambda(x)$ for all $f \in \mathrm{L}^{1}\left(\mathbb{R}^{N}\right), x \in X$;

4) $f x=0$ if $(\operatorname{supp} \widehat{f}) \cap \Lambda(x)=\varnothing$, where $f \in \mathrm{L}^{1}\left(\mathbb{R}^{N}\right), \mathrm{L}^{1}\left(\mathbb{R}^{N}\right)$; 
5) $f x=x$ if the set $\Lambda(x)$ is compact and $\widehat{f}=1$ in some neighbourhood of the set $\Lambda(x)$, where $x \in X, f \in \mathrm{L}^{1}\left(\mathbb{R}^{N}\right)$;

6) if $\Lambda(x)=\left\{\lambda_{1}, \ldots, \lambda_{n}\right\}$ is a finite set for a vector $x \in \mathcal{X}$, then it is represented as $x=$ $x_{1}+\cdots+x_{n}$, where the vectors $x_{k}, 1 \leqslant k \leqslant n$, possess the properties $\Lambda\left(x_{k}\right)=\left\{\lambda_{k}\right\}$, $T(\tau) x_{k}=\mathrm{e}^{\mathrm{i} \lambda_{k} \tau} x_{k}, \tau \in \mathbb{R}^{\mathbb{N}}, f x_{k}=T\left(f_{k}\right) x_{k}=\widehat{f}\left(\lambda_{k}\right) x_{k}, 1 \leqslant k \leqslant n ;$

7) if a subset $X_{0} \subset X_{1}$ is dense in $X_{1}$, then $\Lambda\left(X_{1}\right)=\overline{\bigcup_{x \in X_{0}} \Lambda(x)}$. If $x=\lim _{n \rightarrow \infty} x_{n}$, then $\Lambda(x) \subset \overline{\bigcup_{n \geqslant 1} \Lambda\left(x_{n}\right)}$.

Remark 1. Property 6) for a function $x$ in $\mathrm{L}^{1}\left(\mathbb{R}^{N}\right)$-modulus $\mathrm{C}_{\mathrm{b}}\left(\mathbb{R}^{N}\right)$ means that it can be represented as a trigonometric polynomial

$$
x(\tau)=\alpha_{1} \mathrm{e}^{\mathrm{i}\left(\lambda_{1}, \tau\right)}+\cdots+\alpha_{n} \mathrm{e}^{\mathrm{i}\left(\lambda_{n}, \tau\right)}, \quad \tau \in \mathbb{R}^{N}, \alpha_{k} \in \mathbb{C} \backslash\{0\}, 1 \leqslant k \leqslant n .
$$

The Beurling spectrum of this function is a set $\Lambda(x)=\left\{\lambda_{1}, \ldots, \lambda_{n}\right\}$.

Remark 2. The definition of the Beurling spectrum implies the identity

$$
\Lambda\left(x \mathrm{e}_{\lambda}\right)=\{\lambda\}+\Lambda(x)=\{\lambda+\mu \mid \mu \in \Lambda(x)\}
$$

for all $\lambda \in \mathbb{R}^{N}, x \in \mathrm{C}_{\mathrm{b}}$ and each non-zero function $x$ in $\mathrm{C}_{\mathrm{b}}$.

In what follows we employ essentially the Loomis theorem [23]; its vector analogue was proved in paper [15]. It was proved for the functions defined on a locally compact Abelian group.

Theorem 10 (Loomis). Let the Beurling spectrum $\Lambda(x)$ of a function $x$ in $\mathrm{C}_{\mathrm{b}, \mathrm{u}}\left(\mathbb{R}^{N}\right)$ is at most countable. Then the function $x$ is Bohr almost periodic; $x \in \operatorname{AP}\left(\mathbb{R}^{N}\right)$.

Besides the algebra $\widehat{\mathrm{L}^{1}}\left(\mathbb{R}^{N}\right)$, we consider the algebra

$$
\begin{gathered}
\widehat{\mathrm{L}_{\text {loc }}^{1}}\left(\mathbb{R}^{N}\right)=\left\{F: \mathbb{R}^{N} \rightarrow \mathbb{C} \mid \widehat{f} F \in \widehat{\mathrm{L}^{1}}\left(\mathbb{R}^{N}\right) \text { for each function } f \in \mathrm{L}^{1}\left(\mathbb{R}^{N}\right)\right. \\
\text { with a compact support supp } \widehat{f}\} .
\end{gathered}
$$

Remark 3. The inclusion holds:

$$
\mathcal{P}\left(\mathbb{R}^{N}\right) \subset \widehat{\mathrm{L}_{\text {loc }}^{1}}\left(\mathbb{R}^{N}\right)
$$

Remark 4. A Banach algebra $\mathrm{L}^{1}\left(\mathbb{R}^{N}\right)$ is regular [2], 3], [4], 24], 25], that is, for each open covering $\mathbb{R}^{N}$ of form $\mathbb{R}^{N}=U_{1} \cup \cdots \cup U_{n} \cup U_{\infty}$, where $\overline{U_{k}}, 1 \leqslant k \leqslant n$, are compact sets, there exist functions $f_{1}, \ldots, f_{n}$ in the algebra $\mathrm{L}^{1}\left(\mathbb{R}^{N}\right)$ such that

1) $\operatorname{supp} \widehat{f}_{k} \subset U_{k}, 1 \leqslant k \leqslant n$;

2) $\operatorname{supp}\left(\mathbf{1}-\widehat{f}_{1}-\cdots-\widehat{f}_{n}\right) \subset U_{\infty}$;

where $\mathbf{1}(\lambda)=\lambda, \lambda \in \mathbb{R}^{N}$, is the function identically equalling to one.

The next statement [2], [4], 24], 25] will be employed essentially in the proof of the main results.

Theorem 11 (Wiener). Let $\varphi \in \widehat{\mathrm{L}_{\text {loc }}^{1}}\left(\mathbb{R}^{N}\right)$ and $\varphi$ belongs to the algebra $\widehat{\mathrm{L}^{1}}\left(\mathbb{R}^{N}\right)$ at infinity, that is, there exists a number $R>0$ and a function $\varphi_{\infty} \in \widehat{\mathrm{L}^{1}}\left(\mathbb{R}^{N}\right)$ such that $\varphi(\lambda)=\varphi_{\infty}(\lambda)$ for all $\lambda \in \mathbb{R}^{N}$ satisfying the condition $|\lambda|>R$. Then $\varphi \in \widehat{\mathrm{L}^{1}}\left(\mathbb{R}^{N}\right)$.

Lemma 4. Let the function $\varphi \in \widehat{\mathrm{L}_{\text {loc }}^{1}}\left(\mathbb{R}^{N}\right)$ possesses the following property: there exists a number $R>0$ and a function $\widehat{\psi} \in \widehat{\mathrm{L}^{1}}\left(\mathbb{R}^{N}\right)$ such that $|\widehat{\psi}(\lambda)| \leqslant \frac{1}{3}$ for $|\lambda|>R$ and $\varphi(\lambda)=\frac{\widehat{\varphi_{1}}(\lambda)}{1-\widehat{\psi}(\lambda)}$, $|\lambda|>R$, for some function $\widehat{\varphi_{1}} \in \widehat{\mathrm{L}^{1}}\left(\mathbb{R}^{N}\right)$. Then $\widehat{\varphi} \in \widehat{\mathrm{L}^{1}}\left(\mathbb{R}^{N}\right)$. 
Proof. In view of the Wiener theorem 11, it is sufficient to establish a local belonging of the function $\varphi$ at infinity to the algebra $\widehat{\mathrm{L}^{1}}\left(\mathbb{R}^{N}\right)$. We consider the function $\widehat{\psi_{1}} \in \widehat{\mathrm{L}^{1}}\left(\mathbb{R}^{N}\right)$ with the properties: $\widehat{\psi_{1}}(\lambda)=\varphi(\lambda),|\lambda| \geqslant R, \max _{\lambda \in \mathbb{R}^{N}}\left|\widehat{\psi_{1}}(\lambda)\right| \leqslant \frac{1}{2}$. Then the spectrum $\sigma\left(\psi_{1}\right)$ of the function $\psi_{1}$ as of an element in the Banach algebra $\mathrm{L}^{1}\left(\mathbb{R}^{N}\right)$ [4], [5] coincides with the set $\widehat{\psi_{1}}\left(\mathbb{R}^{N}\right)$ and this is why it lies in the circle $\left\{\lambda \in \mathbb{C}|| \lambda \mid \leqslant \frac{1}{2}\right\}$. We represent the function $\frac{1}{1-\widehat{\psi_{1}}}$ as $\frac{1}{1-\widehat{\psi}_{1}}=\sum_{n=0}^{\infty}{\widehat{\psi_{1}}}^{n}=\mathbf{1}+\widehat{\psi_{2}}$, where $\widehat{\psi_{2}} \in \widehat{\mathrm{L}^{1}}\left(\mathbb{R}^{N}\right)$. Therefore, the function $\frac{\widehat{\varphi_{1}}}{1-\widehat{\psi}_{1}}$ belongs to the algebra $\widehat{\mathrm{L}^{1}}\left(\mathbb{R}^{N}\right)$, and this is why the function $\varphi$ belongs to the algebra $\mathrm{L}^{1}\left(\mathbb{R}^{N}\right)$ at infinitey.

In the algebra $\widehat{\mathrm{L}^{1}}\left(\mathbb{R}^{N}\right)$ we consider a bounded approximative unit $\left(f_{n}\right)$, which is constructed as follows.

We consider a function $f_{0}$ in the algebra $\mathrm{L}^{1}(\mathbb{R})$, for which $\widehat{f}(0)=1$ and $\operatorname{supp} \widehat{f}_{0} \subset[-1,1]$. For instance, this can be a function $f_{0}$ with $\widehat{f}_{0}(\lambda)=(1-|\lambda|) \chi_{[-1,1]}(\lambda), \lambda \in \mathbb{R}$, where $\chi_{[-1,1]}$ is the characteristic function of the segment $[-1,1]$. We let

$$
\widehat{f_{n}}(\tau)=\prod_{k=1}^{N} \widehat{f}_{0}\left(\frac{1}{n} \tau_{k}\right), \quad \tau=\left(\tau_{1}, \ldots, \tau_{N}\right) \in \mathbb{R}^{N}, n \geqslant 1 .
$$

Then the sequence of the functions

$$
f_{n}(\tau)=\frac{1}{(2 \pi)^{N}} \int_{\mathbb{R}^{N}} \widehat{f}_{n}(\lambda) \mathrm{e}^{\mathrm{i}(\lambda, \tau)} \mathrm{d} \lambda, \quad \tau \in \mathbb{R}^{N}, n \geqslant 1,
$$

forms a bunded approximative unit in the algebra $\mathrm{L}^{1}\left(\mathbb{R}^{N}\right)$.

\section{PROOF OF MAIN RESUltS}

We consider some properties of regular at infinity polynomials. While proving them, we shall employ (11).

Lemma 5. Let $p \in \mathcal{P}_{\text {reg, } \infty}\left(\mathbb{R}^{N}\right)$ and $\Delta$ be closed set possessing the property $\mathrm{Z}(p) \cap \Delta=\varnothing$ $(\operatorname{dist}(\mathrm{Z}(p), \Delta)>0)$. Then $\mathrm{Z}(p)$ is compact and for each two open sets $U, V \in \mathbb{R}^{N}$ possessing the properties: $U \supset \mathrm{Z}(p), \bar{U}$ is compact, $\Delta \subset V, \bar{U} \cap \bar{V}=\varnothing$, there exist functions $g_{0}, g_{1}$ in the algebra $\mathrm{L}^{1}\left(\mathbb{R}^{N}\right)$ such that

1) $\widehat{g_{0}}(\lambda)=\mathbf{1}$ in some neighbourhood of the set $\mathrm{Z}(p)$;

2) $\operatorname{supp} \widehat{g_{0}} \subset U$;

3) $p(\lambda) \widehat{g_{1}}(\lambda)-\widehat{g_{0}}(\lambda)=\mathbf{1}, \lambda \in \mathbb{R}^{N}$;

4) if $\mathrm{Z}(p)=\varnothing$, then the function $\frac{1}{p}$ belongs to the algebra $\widehat{\mathrm{L}^{1}}\left(\mathbb{R}^{N}\right)$ (here $\left.g_{0}=0, g_{1}=\frac{1}{p}\right)$.

Proof. We employ the notations from Definition 7 of a regular at infinity polynomial $p$. In view of the property $\lim _{|\lambda| \rightarrow \infty}\left|\widehat{f}_{0}(\lambda)\right|=0$, by identity $\sqrt{7 \mid}$ we obtain that there exists a number $R \geqslant 0$ such that $\left|f_{0}(\lambda)\right| \leqslant \frac{1}{2}$ for $|\lambda| \geqslant R$. This is why by (7) we get the identity

$$
\frac{1}{p(\lambda)}=\frac{\widehat{f}_{1}(\lambda)}{1-\widehat{f}_{0}(\lambda)}, \quad|\lambda| \geqslant R \text {. }
$$

It follows from Lemma 4 that the function $\frac{1}{p}$ belongs to the algebra $\widehat{\mathrm{L}^{1}}\left(\mathbb{R}^{N}\right)$ at infinity.

We consider the function $\widehat{g_{0}} \in \mathrm{L}^{1}\left(\mathbb{R}^{N}\right)$, for which $\widehat{g_{0}}=\mathbf{1}$ in some compact neighbourhood $U_{0}$ of the set $\mathrm{Z}(p)$ and $\operatorname{supp} \widehat{g_{0}} \subset U$; here we employ the normality of the algebra $\mathrm{L}^{1}\left(\mathbb{R}^{N}\right)$, see [2], 3]. By Wiener theorem 11 , the function $\widehat{g_{1}}=\frac{1-\widetilde{g_{0}}}{p}$ belongs to the algebra $\widehat{\mathrm{L}^{1}}\left(\mathbb{R}^{N}\right)$. The function $g_{0}$ can be also constructed straightforwardly by constructing a smooth function $\widehat{g_{0}}$ 
with prescribed properties. The constructed functions $g_{0}$ and $g_{1}$ possess all mentioned in the formulation properties $1-4$. The proof is complete.

Lemma 6. Let a polynomial $p \in \mathcal{P}\left(\mathbb{R}^{N}\right)$ is represented as $p=p_{1}+p_{2}$, where $p_{1} \in \mathcal{P}_{\text {reg, } \infty}\left(\mathbb{R}^{N}\right)$, and a function $\frac{p_{2}}{p_{1}}$ belongs to the algebra $\widehat{\mathrm{L}^{1}}\left(\mathbb{R}^{N}\right)$ at infinity. Then $p \in \mathcal{P}_{\text {reg, }, \infty}\left(\mathbb{R}^{N}\right)$.

The statement of the lemma follows Wiener theorem 11.

Corollary 1. A polynomial $p_{3}: \mathbb{R}^{n} \rightarrow \mathbb{C}$ (see Example 3 after Definition 7 ) employed for defining differential operators studied in paper [1] is regular at infinity.

In fact, this statement was established in paper [1].

Proof of Lemma 1. Let a sequence of the functions $\left(x_{n}, y_{n}\right)$ in the graph

$$
\Gamma\left(\mathcal{A}_{p, \mathcal{F}}\right)=\left\{(x, y) \mid x \in \mathrm{D}\left(\mathcal{A}_{p, \mathcal{F}}\right), y=\mathcal{A}_{p, \mathcal{F}}\right\} \subset \mathcal{F} \times \mathcal{F}
$$

of the operator $\mathcal{A}_{p, \mathcal{F}}$ converge to $\left(x_{0}, y_{0}\right)$ in the Banach space $\mathcal{F} \times \mathcal{F}$ equipped by the norm $\|(x, y)\|=\|x\|+\|y\|, x, y \in \mathcal{F}$. Then for each function $f \in \mathcal{M}$ (supp $\widehat{f}$ is compact) the sequence $\left(f * x_{n}, f * y_{n}\right)$ in $\mathcal{F} \times \mathcal{F}$ possesses the following properties: it converges to $\left(f * x_{0}, f * y_{0}\right) \in \mathcal{F} \times \mathcal{F}$, $\left(f * x_{n}\right) \in \mathrm{D}\left(\mathcal{A}_{p, \mathcal{F}}\right)$ and for $p(f)=\sum_{|\alpha| \leqslant m} a_{\alpha} \mathbb{D}^{\alpha} f$ the identities hold, see (4):

$$
\mathcal{A}_{p, \mathcal{F}}\left(f * x_{0}\right)=p(f) * x_{0}=\lim _{n \rightarrow \infty} p(f) * x_{n}=\lim _{n \rightarrow \infty} f * y_{n}=f * y_{0}, \quad n \geqslant 1 .
$$

It follows from the definition of the operator $\mathcal{A}_{p, \mathcal{F}}$ that $\left(x_{0}, y_{0}\right) \in \Gamma\left(\mathcal{A}_{p, \mathcal{F}}\right)$, that is, $\mathcal{A}_{p, \mathcal{F}}$ is a closed operator.

The above arguing implies also the commutation of the operator $\mathcal{A}_{p, \mathcal{F}}$ with the convolution operators $S(f), f \in \mathcal{M}$. Since the subalgebra $\mathcal{M}$ is dense in the algebra $\mathrm{L}^{1}\left(\mathbb{R}^{N}\right)$, the proven closedness of the operator $\mathcal{A}_{p, \mathcal{F}}$ implies that it commutes with each operator $S(f), f \in \mathrm{L}^{1}\left(\mathbb{R}^{N}\right)$.

Let us prove the commutation of the translation operators $S(\tau), \tau \in \mathbb{R}^{N}$, of the functions in $\mathcal{F}$ with the operator $\mathcal{A}_{p, \mathcal{F}}$. This commutation property is equivalent to the property $(S(\tau) x, S(\tau) y) \in \Gamma\left(\mathcal{A}_{p, \mathcal{F}}\right)$ for each $\tau \in \mathbb{R}^{N}$ and each $(x, y) \in \Gamma\left(\mathcal{A}_{p, \mathcal{F}}\right)$. If $f \in \mathcal{M}$, then $S(\tau) f \in \mathcal{M}$ and this is why $(f * S(\tau) x, f * S(\tau) y) \in \Gamma\left(\mathcal{A}_{p, \mathcal{F}}\right)$, that is, $(S(\tau) x, S(\tau) y) \in \Gamma\left(\mathcal{A}_{p, \mathcal{F}}\right)$. The proof is complete.

Corollary 2. $\operatorname{Ker} \mathcal{A}_{p, \mathcal{F}}$ is a closed submodulus in $\mathcal{F}$.

We recall that the main statements of the paper were obtained by employing the set $\mathrm{Z}(p)$ of zeroes of the polynomial $p$ defined by identity (6). As a rule, we employ formula (1) for the considered polynomial $p$.

While studying the kernel $\operatorname{Ker} \mathcal{A}_{p, \mathcal{F}}$ of the operator $\mathcal{A}_{p, \mathcal{F}}$, we employ essentially the next lemma.

Lemma 7. Let a function $x$ belongs to the kernel $\operatorname{Ker} \mathcal{A}_{p, \mathcal{F}}$ of the operator $\mathcal{A}_{p, \mathcal{F}}$. Then its Beurling spectrum $\Lambda(x)$ obeys the inclusion

$$
\Lambda(x) \subset \mathrm{Z}(p) \cap \Lambda(\mathcal{F}) \subset \mathrm{Z}(p) .
$$

Proof. Assume that a vector $\lambda_{0}$ in $\mathbb{R}^{N}$ does not belong to the set $\mathrm{Z}(p)$. Thus, $p\left(\lambda_{0}\right) \neq 0$. We consider the function $f$ in $\mathcal{M}$ such that $\widehat{f}\left(\lambda_{0}\right) \neq 0$ and $(\operatorname{supp} \widehat{f}) \cap \mathrm{Z}(p)=\varnothing$. By Corollary 2 we obtain that $f * x \in \mathrm{D}\left(\mathcal{A}_{p, \mathcal{F}}\right)$. The identities

$$
0=f * \mathcal{A}_{p, \mathcal{F}} x=\mathcal{A}_{p, \mathcal{F}}(f * x)=g * x=0
$$


hold, where $g=\sum_{|\alpha| \leqslant m} a_{\alpha} \mathbb{D}^{\alpha} f$. Since $\widehat{g}\left(\lambda_{0}\right)=\widehat{p}\left(\lambda_{0}\right) \widehat{f}\left(\lambda_{0}\right) \neq 0$, Definition 5 of the Beurling spectrum implies that $\lambda_{0} \notin \Lambda(x)$. This is why $\Lambda(x) \subset \mathrm{Z}(p)$. Taking into consideration that $\Lambda(x) \subset \Lambda(\mathcal{F})$, we arrive at (14). The proof is complete.

Proof of Theorems 1 and 3 . Since the operator $\mathcal{A}_{p, \mathcal{F}}$ is closed, its kernel is closed. Lemma 1 implies the commutation of the operator $\mathcal{A}_{p, \mathcal{F}}$ with the convolution operators $S(f), f \in \mathrm{L}^{1}$, and translation operators $S(\tau), \tau \in \mathbb{R}^{N}$. This is why $S(f) \operatorname{Ker} \mathcal{A}_{p, \mathcal{F}} \subset \operatorname{Ker} \mathcal{A}_{p, \mathcal{F}}, f \in \mathrm{L}^{1}$, $S(\tau) \operatorname{Ker} \mathcal{A}_{p, \mathcal{F}} \subset \operatorname{Ker} \mathcal{A}_{p, \mathcal{F}}, \tau \in \mathbb{R}^{N}, S(f) \operatorname{Im} \mathcal{A}_{p, \mathcal{F}} \subset \operatorname{Im} \mathcal{A}_{p, \mathcal{F}}, f \in \mathrm{L}^{1}$, and $S(\tau) \operatorname{Im} \mathcal{A}_{p, \mathcal{F}} \subset \operatorname{Im} \mathcal{A}_{p, \mathcal{F}}$, $\tau \in \mathbb{R}^{N}$. Thus, $\operatorname{Ker} \mathcal{A}_{p, \mathcal{F}}$ is a closed submodulus in $\mathcal{F}$ and $\operatorname{Im} \mathcal{A}_{p, \mathcal{F}}$ is a submodulus in $\mathcal{F}$. Lemma 7 yields the inclusion $\operatorname{Ker} \mathcal{A}_{p, \mathcal{F}} \subset \mathcal{F}(\Delta)$, where $\Delta=\mathrm{Z}(p)$. The proof is complete.

Proof of Theorem 2. Lemma/7implies the countability of the set $\Lambda(x)$ if $x \in \operatorname{Ker} \mathcal{A}_{p, \mathcal{F}}$. Since $x \in$ $\mathrm{C}_{\mathrm{b}, \mathrm{u}}\left(\mathbb{R}^{N}\right)$, by Loomis theorem 10 it is almost periodic. Since the set $\Lambda\left(\operatorname{Ker} \mathcal{A}_{p, \mathcal{F}}\right) \subset \mathrm{Z}(p) \cap \Lambda(\mathcal{F})$ is at most countable, by the approximation theorem for almost periodic functions [7] we infer that the submodulus $\operatorname{Ker} \mathcal{A}_{p, \mathcal{F}} \subset \mathrm{C}_{\mathrm{b}, \mathrm{u}}\left(\mathbb{R}^{N}\right)$ is close. Thus, we have established properties 1) and 2).

If $\mathcal{F}=\mathrm{C}_{0}$, thanks to $\operatorname{AP}\left(\mathbb{R}^{N}\right) \cap \mathrm{C}_{0}=\{0\}$, we obtain $\operatorname{Ker} \mathcal{A}_{p, \mathcal{F}}=\{0\}$.

A finite dimension of the kernel Ker $\mathcal{A}_{p, \mathcal{F}}$ under the condition of a finiteness of the set $\mathrm{Z}(p) \cap$ $\Lambda(\mathcal{F})$ is implied by Property 6$)$ in Lemma 3 and Remark 1.

The result of paper [26] imply that the subspace $\operatorname{AP}\left(\mathbb{R}^{N}\right) \cap \mathrm{C}_{\mathrm{sl}}\left(\mathbb{R}^{N}\right)$ is either zero or consists of constant functions. The proof is complete.

In the proof of Lemma 2 and other theorems we shall make use of the following lemma.

Lemma 8. Let $\mathcal{F}$ be a homogeneous space with a compact Beurling spectrum $\Delta=\Lambda(\mathcal{F})$. Then for each polynomial $p \in \mathcal{P}\left(\mathbb{R}^{N}\right)$ of form (1), the operator $\mathcal{A}_{p, \mathcal{F}}$ is bounded and can be represented as

$$
\mathcal{A}_{p, \mathcal{F}} x=\left(\sum_{|\alpha| \leqslant m} a_{\alpha} \mathbb{D}^{\alpha} f\right) * x, \quad x \in \mathcal{F},
$$

where $f$ is an arbitrary function from the algebra $\mathcal{M} \subset \mathrm{L}^{1}\left(\mathbb{R}^{N}\right)$ such that $\widehat{f}=1$ in some neighbourhood of the set $\Delta$. The estimate holds $\left\|\mathcal{A}_{p, \mathcal{F}}\right\| \leqslant\|g\|_{1}$, where $g=\sum_{|\alpha| \leqslant m} a_{\alpha} \mathbb{D}^{\alpha} f$.

Proof. It follows from Property 5) of Lemma 3 that $f * x=x$ for each function $f$ obeying the assumptions of the lemma. Then representation (15) follows the definition if the operator $\mathcal{A}_{p, \mathcal{F}}$. The proof is complete.

Thus, the spectral subspace $\mathcal{F}(\Delta)$ is an invariant subspace for the operators $\mathcal{A}_{p, \mathcal{F}}, p \in \mathcal{P}\left(\mathbb{R}^{N}\right)$. Proof of Lemma 2. Let $x$ be a vector in $\mathrm{D}\left(\mathcal{A}_{p, \mathcal{F}}\right)$ and $y=\mathcal{A}_{p, \mathcal{F}} x$. It follows from the definition $\mathcal{A}_{p, \mathcal{F}}$ that $f * x \in \mathrm{D}\left(\mathcal{A}_{p, \mathcal{F}}\right)$ for each function $f$ in the subalgebra $\mathcal{M} \subset \mathrm{L}^{1}\left(\mathbb{R}^{N}\right)$ and $f * y=\mathcal{A}_{p, \mathcal{F}}(f * x)$. If $\lambda_{0} \notin \Lambda(x)$, then we consider a function $f \in \mathcal{M}$ such that $\widehat{f}\left(\lambda_{0}\right) \neq 0$ and $(\operatorname{supp} \widehat{f}) \cap \Lambda(x)=\varnothing$. Then $f * x=0$, see Property 4 of Lemma 3, and therefore, $f * y=0$, that is, $\lambda_{0} \notin \Lambda(y)$. Thus, $\Lambda(y) \subset \Lambda(x)$ and we have completed the proof of inclusion (5).

Proof of Theorem 4. We first prove Property 1). Let a compact set $\Delta$ in $\mathbb{R}^{N}$ obey $\Delta \cap \mathrm{Z}(p)=\varnothing$. The regularity of the algebra $L^{1}\left(\mathbb{R}^{N}\right)$ implies (see [24]) the existence of a function $f \in \mathcal{M}$ such that $p \widehat{f}=1$ in some neighbourhood of the compact set $\Delta$.

Let $y$ be an arbitrary function in the spectral modulus $\mathcal{F}(\Delta)$ and $x=f * y$. The vector $x$ has a compact Beurling spectrum $\Lambda(x)$, and this is why $x \in \mathrm{D}\left(\mathcal{A}_{p, \mathcal{F}}\right)$ and the identity holds:

$$
\mathcal{A}_{p, \mathcal{F}} x=\left(\sum_{|\alpha| \leqslant m} a_{\alpha} \mathbb{D}^{\alpha} f\right) * y=g * y,
$$


where $\widehat{g}=p \widehat{f}=\mathbf{1}$ in some neighbourhood of the set $\Lambda(y) \subset \Delta$. This is why Property 5) of Lemma 3 implies $g * y=y$, that is, by 16 we obtain $\mathcal{A}_{p, \mathcal{F}} x=y$. Thus, we have established the inclusion $\mathcal{F}(\Delta) \subset \operatorname{Im} \mathcal{A}_{p, \mathcal{F}}$.

We proceed to proving Property 2). Due to the condition $\mathrm{Z}(p)=\varnothing$, the proven first property implies that each spectral submodulus $\mathcal{F}(\Delta)$, where $\Delta$ is a compact set, is contained in $\operatorname{Im} \mathcal{A}_{p, \mathcal{F}}$. Let $y$ be an arbitrary function in $\mathcal{F} \subset \mathrm{C}_{\mathrm{b}, \mathrm{u}}$. We consider an approximative unit $\left(f_{n}\right)$ in the algebra $L^{1}\left(\mathbb{R}^{N}\right)$ constructed in the end of Section 2. Then, see [2], [3], [16], [17], $\lim _{n \rightarrow \infty} f_{n} * y=y$. Since $\Lambda\left(f_{n} * y\right) \subset \operatorname{supp} \widehat{f}_{n}, n \geqslant 1$, are compact set, then $f_{n} * y \in \operatorname{Im} \mathcal{A}_{p, \mathcal{F}}, n \geqslant 1$, by Property 1$)$. Therefore, $y \in \operatorname{Im} \mathcal{A}_{p, \text { F }}$.

We proceed to proving Property 3). Let $y$ be an arbitrary function in $\mathrm{C}_{0}\left(\mathbb{R}^{N}\right)$. For the considered approximative unit $\left(f_{n}\right)$ in the algebra $L^{1}\left(\mathbb{R}^{N}\right)$ (see $\sqrt{12}$ ), $(13)$ ), the sequence of the functions $y_{n}=f_{n} * y, n \geqslant 1$, possesses the following properties: $\lim _{n \rightarrow \infty} y_{n}=y$ thanks to the uniform continuity of the function $y$ and $\Delta_{n}=\Lambda\left(y_{n}\right) \subset \operatorname{supp} \widehat{f}_{n} \subset[-1,1]^{N}, n \geqslant 1$, are compact sets. This is why it is sufficient to show that each function $z \in \mathrm{C}_{0}\left(\mathbb{R}^{N}\right)$ with a compact spectrum $\Lambda(z)$ is in the closure of the image $\operatorname{Im} \mathcal{A}_{p, \mathrm{C}_{0}}$.

First we assume that $\Lambda(z) \cap \mathrm{Z}(p)=\left\{\lambda_{0}\right\}$ is a single point set. We consider a sequence of the functions $\left(\varphi_{n}\right)$ in the algebra $L^{1}\left(\mathbb{R}^{N}\right)$ with the following properties: $\sup _{n \geqslant 1}\left\|\varphi_{n}\right\|<\infty, \widehat{\varphi_{n}}=0$ in some neighbourhood $V_{n}$ of the point $\lambda_{0}$ and $\lim _{n \rightarrow \infty} \varphi_{n} * g=0$ for each function $g$ in the algebra $\mathrm{L}^{1}\left(\mathbb{R}^{N}\right)$ obeying the identity $\widehat{g}\left(\lambda_{0}\right)=0$. In works [2], 3], [15], [16], [26], [27], this sequence was called $\lambda_{0}$-sequence. It was also established there that for a function $x \in \mathrm{C}_{\mathrm{b}, \mathrm{u}}\left(\mathbb{R}^{N}\right)$, there exiss the uniform in $\tau \in \mathbb{R}^{N}$ limit

$$
\lim _{n \rightarrow \infty} \frac{1}{n^{N}} \int_{0}^{n} \ldots \int_{0}^{n} x(s+\tau) \mathrm{e}^{-\mathrm{i}\left(\lambda_{0}, s+\tau\right)} \mathrm{d} s=x_{0}(\tau), \quad \tau \in \mathbb{R}^{N},
$$

then the limit $\lim _{n \rightarrow \infty} \varphi_{n} * x$ exists as well and they coincide. Such result follows general ergodic theorems [2], 33. The first limit $x_{0}$ for $x=z$ is the zero function since the function $z$ belongs to the space $\mathrm{C}_{0}\left(\mathbb{R}^{N}\right)$ ), and hence $\lim _{n \rightarrow \infty} \varphi_{n} * z=0$. Let $f$ be a function in $\mathrm{L}^{1}\left(\mathbb{R}^{N}\right)$ such that $\widehat{f}=\mathbf{1}$ in some neighbourhood of the compact set $\Lambda(z)$. Then $f * z=z$ by Property 5 of Lemma 3 . Let $z_{n}=z-\varphi_{n} * z=f *\left(z-\varphi_{n} * z\right), n \geqslant 1$. By Property 3) of Lemma 3 we obtain that $\Lambda\left(z_{n}\right) \subset \operatorname{supp}\left(\widehat{f}-\widehat{f} \widehat{\varphi_{n}}\right) \cap \Lambda(z), n \geqslant 1$. Therefore, $\lambda_{0} \notin \Lambda\left(z_{n}\right)$ and $\Lambda\left(z_{n}\right) \cap \mathrm{Z}(p)=\varnothing$. This is why Property 1 implies $z \in \overline{\operatorname{Im} \mathcal{A}_{p, \mathrm{C}_{0}}}$. If $\Lambda(z) \cap \mathrm{Z}(p)=\left\{\lambda_{1}, \ldots, \lambda_{n}\right\}$ is a finite zet, we represent the function $z$ as $z=f_{1} * z+\cdots+f_{n} * z$, where the functions $f_{k}, 1 \leqslant k \leqslant n$, are in the algebra $\mathrm{L}^{1}(\mathbb{Z})$ and they possess the following properties: the function $f=f_{1}+\cdots+f_{n}$ has a Fourier transform $\widehat{f}=\mathbf{1}$ in some neighbourhood of the compact set $\Lambda(z), \widehat{f}_{k}\left(\lambda_{k}\right)=1,1 \leqslant k \leqslant n$, $\lambda_{k} \notin \operatorname{supp} \widehat{f}_{n}$ for $k \neq n$. Then $\Lambda\left(z_{k}\right) \cap \mathrm{Z}(p)=\left\{\lambda_{k}\right\}, 1 \leqslant k \leqslant n$, and thanks to the above proven facts, $z_{k} \in \overline{\operatorname{Im} \mathcal{A}_{p, \mathrm{C}_{0}}}, 1 \leqslant k \leqslant n$. Therefore, $z \in \overline{\operatorname{Im} \mathcal{A}_{p, \mathrm{C}_{0}}}$.

Let us prove Property 4$)$. Let $y=\mathcal{A}_{p, \mathrm{C}_{\mathrm{sl}}} x$, where $x \in \mathrm{D}\left(\mathcal{A}_{p, \mathrm{C}_{\mathrm{sl}}}\right)$. We consider the sequence of functions $\left(\varphi_{n}\right)$ in the algebra $\mathrm{L}^{1}\left(\mathbb{R}^{N}\right)$ introduced in the proof of Property 3 and the sequence $y_{n}=\varphi_{n} * y, n \geqslant 1$, in $\mathrm{C}_{\mathrm{sl}}\left(\mathbb{R}^{N}\right)$. It follows from the results of paper [26] that $y-y_{0} \in \mathrm{C}_{0}\left(\mathbb{R}^{N}\right)$, $n \geqslant 1$. Lemma 7 and Definition 4 imply that $y_{n}=\varphi_{n} * y=\mathcal{A}_{p, \mathrm{C}_{\mathrm{sl}}}\left(\varphi_{n} * x\right)=p\left(\varphi_{n}\right) * x$, where $p\left(\varphi_{n}\right)=\sum_{|\alpha| \leqslant m} a_{\alpha} \mathbb{D}^{\alpha} \varphi_{n}, \widehat{p\left(\varphi_{n}\right)}=p \widehat{\varphi_{n}}$, and each of the functions $p \widehat{\varphi_{n}}, n \geqslant 1$, can be represented as $\widehat{g} \widehat{\varphi_{n}}$, where $\widehat{g}=p \widehat{f}$ and $f \in \mathrm{L}^{1}\left(\mathbb{R}^{N}\right), \widehat{f}=\mathbf{1}$ in the neighbourhood of $\operatorname{supp} \widehat{\varphi_{1}}$. This is why, in view of the property $\widehat{g}(0)=0$, we obtain that $\lim _{n \rightarrow \infty}\left\|p\left(\varphi_{n}\right)\right\|_{1}=0$. Therefore, $\lim _{n \rightarrow \infty}\left\|y_{n}\right\|=0$ and this implies $y \in \mathrm{C}_{0}\left(\mathbb{R}^{N}\right)$. The proof is complete. 
Lemma 9. Let $p \in \mathcal{P}_{\text {reg, } \infty}\left(\mathbb{R}^{N}\right)$ and $g_{0}, g_{1}$ be functions in the algebra $\mathrm{L}^{1}\left(\mathbb{R}^{N}\right)$ obeying the assumptions 5 . Then the following identities hold

$$
\operatorname{Im} S\left(g_{1}\right)=\mathrm{D}\left(\mathcal{A}_{p, \mathcal{F}}\right) \subset \mathrm{C}_{\mathrm{b}, \mathrm{u}}\left(\mathbb{R}^{N}\right), \quad \mathcal{A}_{p, \mathcal{F}} S\left(g_{1}\right)-S\left(g_{0}\right)=I \in \text { End } \mathcal{F}
$$

and

$$
g_{1} * \mathcal{A}_{p, \mathcal{F}} x-g_{0} * x=x, \quad x \in \mathrm{D}\left(\mathcal{A}_{p, \mathcal{F}}\right) .
$$

Proof. Let $x$ be a function in $\mathrm{D}\left(\mathcal{A}_{p, \mathcal{F}}\right)$ and $f \in \mathcal{M}$. Since the operator $S(f)$ commutes with the operator $\mathcal{A}_{p, \mathcal{F}}$, see Lemma 1. we have $S(f) x \in \mathrm{D}\left(\mathcal{A}_{p, \mathcal{F}}\right)$, and then the function $y=S(f)\left(\mathcal{A}_{p, \mathcal{F}} S\left(g_{1}\right)-S\left(g_{0}\right)\right) x$ can be represented as $y=\mathcal{A}_{p, \mathcal{F}} S\left(f * g_{1}\right)-S\left(f * g_{0}\right) x=g * x$, where $\widehat{g}(\lambda)=p(\lambda) \widehat{f}(\lambda) \widehat{g}_{1}(\lambda)-\widehat{f}(\lambda) \widehat{g_{0}}(\lambda)=0, \lambda \in \mathbb{R}^{N}$. Thus, $S(f) y=0$ for each function $f$ in $\mathcal{M}$. The property $\overline{\mathcal{M}}=\mathrm{L}^{1}\left(\mathbb{R}^{N}\right)$ implies that $f * y=0$ for each function $f$ in the algebra $\mathrm{L}^{1}\left(\mathbb{R}^{N}\right)$ and this is why $y=0$. This completes the proof of identity (17). The proven fact implies $\operatorname{Im} S\left(g_{1}\right) \subset \mathrm{D}\left(\mathcal{A}_{p, \mathcal{F}}\right)$.

In the same way we establish identity (18). It follows from identities (17) and (18) that $\mathrm{D}\left(\mathcal{A}_{p, \mathcal{F}}\right)=\operatorname{Im} S\left(g_{1}\right)$. Since the functions in the image of the convolution operator are uniformly continuous, we arrive at $\mathrm{D}\left(\mathcal{A}_{p, \mathcal{F}}\right) \subset \mathrm{C}_{\mathrm{b}, \mathrm{u}}$. The proof is complete.

Lemma 10. If the operator $\mathcal{A}_{p, \mathcal{F}}$ is invertible, it is invertible on all spectral submoduli in $\mathcal{F}$ and the property holds:

$$
\mathrm{Z}(p) \cap \Lambda(\mathcal{F})=\varnothing
$$

Proof. Assume that condition (10) is not satisfied and let $\lambda_{0} \in \mathrm{Z}(p) \cap \Lambda(\mathcal{F})$. We consider any compact neighbourhood $\Delta$ containing the point $\lambda_{0}$ and the restriction $\mathcal{A}_{\Delta}=\mathcal{A}_{p, \mathcal{F}} \mid \mathcal{F}(\Delta)$ of the operator $\mathcal{A}_{p, \mathcal{F}}$ on the spectral subspace $\mathcal{F}(\Delta)$. Let us prove that $\lambda_{0} \in \Lambda(\mathcal{F}(\Delta))$ and therefore, $\mathcal{F}(\Delta)$ is a non-zero subspace in $\mathcal{F}$. In order to do this, we consider a function $f$ in the algebra $\mathrm{L}^{1}\left(\mathbb{R}^{N}\right)$ with the properties $\widehat{f}\left(\lambda_{0}\right) \neq 0$ and supp $\widehat{f} \subset \Delta$. Then $f * x \in \mathcal{F}(\Delta), \lambda_{0} \in \Lambda(f * x)$. Hence, $0 \neq f x \in \mathcal{F}(\Delta)$.

According Lemma 8, the operator $\mathcal{A}_{\Delta}$ is bounded $\left(\mathcal{A}_{\Delta} \in \operatorname{End} \mathcal{F}(\Delta)\right)$, and by the proven above it is invertible and the inverse $\mathcal{A}_{\Delta}^{-1} \in \operatorname{End} \mathcal{F}(\Delta)$ is the restriction of the operator $\mathcal{A}_{p, \mathcal{F}}^{-1}$ on the spectral subspace $\mathcal{F}(\Delta)$. It follows from Lemma 9 that the operator $\mathcal{A}_{p}^{-1}$ is the convolution operator with the function $g_{1} \in \mathrm{L}^{1}\left(\mathbb{R}^{N}\right)$. To prove this, it is sufficient to choose a function $g_{0}$ so that $\left(\operatorname{supp} \widehat{g_{0}}\right) \cap \Delta=\varnothing$. Since $\widehat{g_{1}}=p$ in some neighbourhood of the set $\Delta$, then $\widehat{g_{1}}\left(\lambda_{0}\right)=$ $p\left(\lambda_{0}\right)=0$. Since the algebra $\mathrm{L}^{1}\left(\mathbb{R}^{N}\right)$ satisfies the Ditkin condition [2], [3], there exists a bounded sequence $\left(\varphi_{n}\right)$ in the algebra $L^{1}\left(\mathbb{R}^{N}\right)$ with the following properties: $\widehat{\varphi_{n}}=0$ in some open neighbourhood $V_{n} \subset \Delta$ of the point $\lambda_{0}$ for each $n \in \mathbb{N}$ and $\lim _{n \rightarrow \infty}\left\|\varphi_{n} * g_{1}\right\|_{1}=0$. We consider the sequence $A_{n} \in$ End $\mathcal{F}(\Delta), n \geqslant 1$, of form

$$
\mathcal{A}_{n}=\mathcal{A}_{\Delta}-S\left(\varphi_{n}\right) \mathcal{A}_{\Delta}=S\left(g_{1}-\varphi_{n} * g_{1}\right), \quad n \geqslant 1 .
$$

This representation implies that

$$
\left\|\mathcal{A}_{\Delta}-\mathcal{A}_{n}\right\|=\left\|S\left(\varphi_{n}\right) S(g)\right\|=\left\|S\left(\varphi_{n} * g_{n}\right)\right\| \rightarrow 0, \quad n \rightarrow \infty .
$$

Let $\Delta_{n}$ be a compact neighbourhood of the point $\lambda_{0}$ contained $V_{n}$. Since the point $\lambda_{0}$ belongs to the set $\Lambda(\mathcal{F}(\Delta))$ and $\lambda_{0} \in \Delta$, then $\mathcal{F}\left(\Delta_{n}\right)$ is a non-zero spectral subspace in $\mathcal{F}$. Since the function $\widehat{g_{1}}-\widehat{\varphi_{n}} \widehat{g_{1}}$ vanishes in the vicinity of the set $\Delta_{n}$, it follows from Property 3 of Lemma 3 that $\{0\} \neq \mathcal{F}\left(\Delta_{n}\right) \subset \operatorname{Ker} \mathcal{A}_{n}$ for each $n \geqslant 1$. Thus, we obtain that the invertible operator $\mathcal{A}_{\Delta}$ is a limit (in the operator norm) of a sequence of non-invertible operators $A_{n}, n \geqslant 1$. This leads us to a contradiction and completes the proof.

We note that Lemma 10 can be proved very easily in the case, when $\mathcal{F}$ contains the subspace $\operatorname{AP}\left(\mathbb{R}^{N}\right)$. 
Proof of Theorem 5. The necessity of condition (8) was established in Lemma 10.

Assume that condition (8) holds. Since the polynomial $p$ is regular at infinity, by Lemma 5 there exist functions $g, g_{0} \in \mathrm{L}^{1}\left(\mathbb{R}^{N}\right)$, for which Conditions 1,2 of Lemma 5 are satisfied. The regularity at infinity of the polynomial $p$ implies that the set of its zeroes $\mathrm{Z}(p)$ is compact. Condition (8) yields that

$$
\operatorname{dist}(\mathrm{Z}(p), \Lambda(\mathcal{F}))=\inf _{\substack{\lambda \in \mathrm{Z}(p) \\ \mu \in \Lambda(\mathcal{F})}}|\lambda-\mu|>0
$$

We turn to Lemma 5, letting $\Delta=\Lambda(\mathcal{F})$, and consider the functions $g_{0}, g_{1} \in \mathrm{L}^{1}\left(\mathbb{R}^{N}\right)$ in this lemma.

Let us prove that the convolution operator $S(g)$ is inverse for the operator $\mathcal{A}_{p, \mathcal{F}}$. The definition of the function $\widehat{g_{1}}$ implies immediately that

$$
\widehat{g_{1}}-\mathbf{1}=\widehat{g_{0}} \in \widehat{\mathrm{L}^{1}}\left(\mathbb{R}^{N}\right),
$$

this is why by Lemma 9 we obtain that $\operatorname{Im} S\left(g_{1}\right)=\mathrm{D}\left(\mathcal{A}_{p, \mathcal{F}}\right)$, and there hold identities (17), (18) in the assumptions of Lemma 9. Since $\widehat{g_{0}}=0$ in some neighbourhood of the set $\Lambda(\mathcal{F})$, then $g_{0} * x=0$ for each function $x$ in $\mathcal{F}$, that is, $S\left(g_{0}\right)$ is a zero operator. Therefore, the operato $\mathcal{A}_{p, \mathcal{F}}$ is invertible and $\mathcal{A}_{p, \mathcal{F}}^{-1}=S(g)$. The proof is complete.

As it was mentioned above, Theorem 6 follows Theorem 5 .

Proof of Theorem 7. The first of the theorem is due to Theorem 5. It implies that the operator $\mathcal{A}_{p}^{-1}$ is of form $(9)$. By this formula we infer that the Fourier series of the periodic function $\mathcal{A}_{p}^{-1} y$ reads as

$$
\left(\mathcal{A}_{p}^{-1} y\right)(\tau) \sim \sum_{k \in \mathbb{Z}} \widetilde{g}\left(\lambda_{k}\right) \mathrm{e}^{\mathrm{i}\left(\lambda_{k}, \tau\right)}=\sum_{k \in \mathbb{Z}} \frac{1}{p\left(2 \pi\left(\frac{k_{1}}{\omega_{1}}, \ldots, \frac{k_{N}}{\omega_{N}}\right)\right)} \mathrm{e}^{\mathrm{i}\left(\lambda_{k}, \tau\right)},
$$

each function $\mathcal{A}_{p}^{-1} y, y \in \mathrm{C}_{\Omega}$, possesses the Fourier series of the form

$$
\left(\mathcal{A}_{p}^{-1} y\right)(\tau) \sim \sum_{k \in \mathbb{Z}^{N}} \widehat{g}\left(\lambda_{k}\right) \mathrm{e}^{\mathrm{i} 2 \pi\left(\lambda_{k}, \tau\right)}=\sum_{k \in \mathbb{Z}^{N}} \frac{1}{p\left(2 \pi\left(\frac{k_{1}}{\omega_{1}}, \ldots, \frac{k_{N}}{\omega_{N}}\right)\right)} \mathrm{e}^{\mathrm{i} 2 \pi\left(\lambda_{k}, \tau\right)}, \quad \tau \in \mathbb{R}^{N},
$$

where $\widetilde{\omega}=\left(\frac{1}{\omega_{1}}, \ldots, \frac{1}{\omega_{N}}\right), \lambda_{k}=\left(\frac{k_{1}}{\omega_{1}}, \ldots, \frac{k_{N}}{\omega_{N}}\right)$. It is straightforward to check, the Fourier series of the function

$$
z(\tau)=\int_{K} G(\tau-s) y(s) \mathrm{d} s, \quad \tau \in \mathbb{R}^{N},
$$

coincides with the above Fourier series of the function $\mathcal{A}_{p}^{-1} y$ and this is why the function $\mathcal{A}_{p}^{-1} y$ coincides with the function $z$. The proof is complete.

Proof of Theorem 8. For each $\lambda_{0} \in \mathbb{C}$ the operator $\mathcal{A}_{p, \mathcal{F}}-\lambda_{0} I$ coincides with the operator $\mathcal{A}_{p_{0}, \mathcal{F}}$, where $p_{0}(\lambda)=p(\lambda)-\lambda_{0}, \lambda \in \mathbb{R}^{N}$. Thanks to Lemma 5, it is clear that the polynomial $p_{0}$, as well as $p$, is regular at infinity and this is why the operator $\mathcal{A}_{p, \mathcal{F}}-\lambda_{0} I$ is invertible if and only if the condition $\lambda_{0} \notin p(\Lambda(\mathcal{F}))$ holds. Thus, we have established identity (8). For a spectrally homogeneous space $\Lambda(\mathcal{F})=\mathbb{R}^{N}$ we have and this is why in this case identity $(8)$ holds true. The proof is complete.

Proof of Theorem 9. Since the set $\Lambda(\mathcal{F})$ has no finite limiting points, the property $p \in$ $\mathcal{P}_{\text {reg, } \infty}\left(\mathbb{R}^{N}\right)$ and Theorem 8 imply that the resolvent set $\rho\left(\mathcal{A}_{p, \mathcal{F}}\right)$ is non-empty. In what follows, without loss of generality, we assume that $\lambda_{0}=0$. Then Theorem 5 implies that the 
inverse operator $\mathcal{A}_{p, \mathcal{F}}^{-1}$ is represented as

$$
\left(\mathcal{A}_{p, \mathcal{F}}^{-1} x\right)=g * x, \quad x \in \mathcal{F}
$$

for some function $g \in \mathrm{L}^{1}\left(\mathbb{R}^{N}\right)$. We consider the aforementioned approximative unit $\left(f_{n}\right)$ in the algebra $\mathrm{L}^{1}\left(\mathbb{R}^{N}\right)$ and a sequence of the operators

$$
A_{n} x=S\left(f_{n}\right) \mathcal{A}_{p, \mathcal{F}}^{-1} x=S\left(f_{n}\right) S(g) x, \quad x \in \mathcal{F}, \quad n \geqslant 1 .
$$

Then $\left\|\mathcal{A}_{p, \mathcal{F}}^{-1}-A_{n}\right\| \leqslant\left\|g-f_{n} * g\right\|_{1} \rightarrow 0, n \rightarrow \infty$.

Let us prove that each of the operators $A_{n}, n \geqslant 1$, is a finite rank operator. The image $\operatorname{Im} S(g)$ of the operator is contained in the subspace $\mathcal{F} \cap \mathrm{C}_{\mathrm{b}, \mathrm{u}}$, and it follows from Properties 2 and 3 of Lemma 3 that $\Lambda(\operatorname{Im} S(g)) \subset \Lambda(\mathcal{F})$, that is, it is either countable or finite set. Therefore, by Loomis theorem $10, \operatorname{Im} S(g) \subset \operatorname{AP}\left(\mathbb{R}^{N}\right)$. Let

$$
\Lambda(\operatorname{Im} S(g))=\left\{\lambda_{k} \mid k \geqslant 1\right\} \subset \mathbb{R}^{N}
$$

where $\lim _{k \rightarrow \infty}\left|\lambda_{k}\right|=\infty$; for the sake of definiteness, we assume that $\Lambda(\mathcal{F})$ is a countable set. By assumption, $\operatorname{supp} \widehat{f}_{n}$ is a compact set and each of the sets $\Lambda\left(\operatorname{Im} A_{n}\right), n \geqslant 1$, is finite and consists of the trigonometric polynomials, see Remark 1 . This is why $\operatorname{Im} A_{n}$ is a finitedimensional subspace in $\mathcal{F}$. Therefore, the operator $\mathcal{A}_{p, \mathcal{F}}^{-1} \in$ End $\mathcal{F}$ is compact as a limit of finite rank operators. The proof is complete.

We note that the main results of the paper are also true for the operators acting in real functions spaces and at that, one can employ the approach developed in papers [18], [28], [29].

\section{BIBLIOGRAPHY}

1. E.M. Mukhamadiev, A.N. Naimov, A.H. Sattorov. Analogue of Bohl theorem for a class of linear partial differential equations // Ufimskij Matem. Zhurn. 9:1, 75-88 (2017). [Ufa Math. J. 9:1, 75-88 (2017).]

2. A.G. Baskakov. Harmonic analysis in Banach moduli and spectral theory of linear operators. Voronezh State Univ. Publ., Voronezh (2016).

3. A. G. Baskakov. Representation theory for Banach algebras, Abelian groups, and semigroups in the spectral analysis of linear operators // Sovrem. Matem. Fundam. Naprav. 9, 3-151 (2004). [J. Math. Sci. 137:4, 4885-5036 (2006).]

4. E. Hewitt, K.A. Ross. Abstract harmonic analysis. Springer-Verlag, Berline (1963).

5. W. Rudin. Fourier analysis on groups. Interscience Publ., New York (1962).

6. A.G. Baskakov, N.B. Uskova. Fourier method for first order differential equations with involution and for groups of operators // Ufimskij Matem. Zhurn. 10:3, 11-34 (2018). [Ufa Math. J. 10:3, 11-34 (2018).]

7. B.M. Levitan. Almost periodic functions. Gostekhizdat, Moscow (1953). (in Russian).

8. B.M. Levitan, V.V. Zhikov. Almost periodic functions and differential equations. Izdat. MGU, Moscow (1978). [Cambridge Univ. Press, Cambridge (1982).]

9. A.G. Baskakov. Analysis of linear differential equations by methods of the spectral theory of difference operators and linear relations // Uspekhi Matem. Nauk. 68:1(409), 77-128 (2013). [Russ. Math. Surv. 68:1, 69-116 (2013).]

10. A.G. Baskakov. Harmonic and spectral analysis of power bounded operators and bounded semigroups of operators on Banach spaces // Matem. Zamet. 98:2, 174-190 (2015). [Math. Notes. 98:2, 164-178 (2015).]

11. A. Baskakov, I. Strukova. Harmonic analysis of functions periodic at infinity // Eurasian Math. J. 7:4, 9-29 (2016).

12. L.I. Ronkin. Introduction to the theory of entire functions of several variables. Nauka, Moscow (1971). [Transl. Math. Monog. 44. Amer. Math. Soc., Providence, R.I. (1974).] 
13. A. G. Baskakov, I. A. Krishtal. Memory estimation of inverse operators // J. Funct. Anal. 267:8, 2551-2605 (2014).

14. Y. Domar. Harmonic analysis based in certain commutative Banach algebras // Acta Math. 96, 1-66 (1956).

15. A.G. Baskakov. Spectral criteria for almost periodicity of solutions of functional equations // Matem. Zamet. 24:2, 195-206 (1978). [Math. Notes. 24:2, 606-612 (1978).]

16. A.G. Baskakov. Harmonic analysis of cosine and exponential operator-valued functions // Matem. Sborn. 124(166):1(5), 68-95 (1984). [Math. USSR-Sb. 52:1, 63-90 (1985).]

17. A.G. Baskakov, I.A. Krishtal. Harmonic analysis of causal operators and their spectral properties // Izv. RAN. Ser. Matem. 69:3, 3-54 (2005). [Izv. Math. 69:3, 439-486 (2005).]

18. E.E. Dikarev. On Bernstein inequality for vectors in Banach spaces // Ufimskij Matem. Zhurn. 5:4, 77-83 (2013). [Ufa Math. J. 5:4, 75-81 (2013).]

19. M. Taylor. Pseudo differential operators. Lecture Notes in Math. 416. Springer-Verlag, Berlin (1974).

20. L. Hörmander. The analysis of linear partial differential operators. II: Differential operators with constant coefficients. Springer, Berlin (1985).

21. S. Mizohata. The theory of partial differential equations. Cambridge University Press, London (1973).

22. Yu.V. Egorov. Linear differential equations of principal type. Fizmatlit, Moscow (1984). [Cont. Soviet Math. Consultants Bureau, New York (1986).]

23. L.H. Loomis. The spectral characterization of a class of almost periodic functions // Ann. Math. 72:2, 362-368 (1960).

24. I.M. Gelfand, D.A. Raikov, G.E. Shilov. Commutative normed rings. Fizmatlit, Moscow (2011). (in Russian).

25. E. Kaniuth. A Course in commutative banach algebras. Springer, New York (2008).

26. A.G. Baskakov, N.S. Kaluzhina. Beurlings theorem for functions with essential spectrum from homogeneous spaces and stabilization of solutions of parabolic equations // Matem. Zamet. 92:5, 643-661 (2012). [Math. Notes. 92:5, 587-605 (2012).]

27. A.G. Baskakov. Spectral synthesis in Banach modules over commutative Banach algebras // Matem. Zamet. 34:4, 776-782 (1983). [Math. Notes. 34:4, 776-782 (1983).]

28. E.E. Dikarev, D.M. Polyakov. Harmonic analysis of nonquasianalytic operators in real Banach space // Vestnik NGU. Ser. Matem. Mekh. Inform. 14:3, 19-28 (2014). [J. Math. Sci. 213:6, 823-831 (2016).]

29. E.E. Dikarev, D.M. Polyakov. Harmonic analysis of some classes of linear operators on a real Banach space // Matem. Zamet. 97:5, 670-680 (2015). [Math. Notes. 97:5, 684-693 (2015).]

Anatoly Grigorievich Baskakov,

Voronezh State University,

Universitetskaya sq. 1,

394018, Voronezh, Russia

E-mail: anatbaskakov@yandex.ru

Egor Evgenievich Dikarev,

Voronezh State University,

Universitetskaya sq. 1,

394018, Voronezh, Russia

E-mail: egor.dikarev@gmail.com 